\title{
Artificial Insemination in Canines: A Review
}

\author{
Jumi Dutta $^{1^{*}}$ and Joli Dutta ${ }^{2}$ \\ ${ }^{1}$ Department of Veterinary Biochemistry, College of Veterinary science, Assam \\ Agricultural University, Guwahati-22, Assam, India \\ ${ }^{2}$ Department of Plant Pathology, Assam Agricultural University, \\ Jorhat- 785013, Assam, India \\ *Corresponding author
}

\begin{abstract}
A B S T R A C T
Artificial Insemination (AI) is the process of collecting semen and depositing it through artificial means into the female reproductive tract of receptive bitch at proper time and place. It is a useful way of decreasing the stress on parent stock who are separated by large geographical distances. Research on AI in the domestic dog, along with other reproductive technologies, proceed worldwide, particularly on sperm survival at freezing and the identification of deleterious components to spermatozoa or fertilization, providing important information for the preservation of wild canidae semen that are currently threatened or endangered. Main indications for $\mathrm{AI}$ in dogs include both medical and breeding-management reasons. As major potential advantage, AI may allow to reduce physical distances, the use of genetically valuable stud dog semen all over the world, fighting the stress of transportation of animals and inbreeding. It is also an important technique whenever physical and behavioural abnormalities in the male or female preventing natural mating. Avoiding direct contact between the male and female, AI also prevents the spread of sexually transmitted diseases, as those originated by Brucella canis or Herpes virus.
\end{abstract}

\section{Keywords}

Artificial

insemination,

Spermatozoa, Stud

dog, Breeding,

Fertilization

\section{Article Info}

Accepted:

20 July 2020

Available Online:

10 August 2020

\section{Introduction}

In Artificial Insemination (AI) the semen is collected manually from a stud male and thereafter deposited (inseminated) in the female so that fertilization can occur in the absence of natural mating. Artificial insemination (AI) has been widely used in cattle for decades, but only in recent years it has found a niche amongst dog breeders. Artificial insemination is an assisted reproductive technique that can be used to compensate for some causes of canine infertility. Many reasons may lead to the request for artificial insemination in dogs. In most cases, inability or unwillingness to copulate naturally or difficulty in achieving or maintaining a successful pregnancy is 
involved in the decision to seek help ${ }^{9}$ It is most frequently carried out by experienced inseminators or by vets in practice. The competence of the operator to perform the procedures is essential to avoid all techniquerelated ethical constraints to the use of $\mathrm{AI}$ in dogs. Before offering canine AI services, practitioners ought to specialised themselves, acquiring profound knowledge of the reproductive physiology and pathology of the species and the skills to collect semen and to inseminate the female without risking animal health or welfare. Ensuring that both the male and female are healthy. The general health of the male and female dog is assessed with a breeding soundness and prebreeding examination, respectively. ${ }^{10}$ Only healthy animals without heritable genetic defects should be considered for artificial insemination. Information concerning possible heritable diseases should be obtained by questioning the owner. A general physical examination should be performed before, not at the time of, insemination to identify any problems. The semen quality should be ascertained well in advance of the insemination procedure. Poor-quality semen may result in an unsuccessful pregnancy or contribute to small litter sizes. $^{2}$

\section{History of AI}

The history of artificial insemination (AI) began with the discovery of spermatozoa in semen by Antony Van Leeuwenhoek in 1677

First AI dates back to 1322 where Arabian horses were artificially bred

It was more than a hundred years later that artificial insemination was accomplished successfully

This first AI was performed by Lazzaro Spallanzani in 1780 by inseminating a bitch which later gave birth to three pups. He is also called as "Father of modern artificial insemination".

1914- G. Amantia, professor of human physiology at University of Rome developed the first artificial vagina for collection of semen from dog.

1937- Danish veterinarians developed the first rectovaginal/cervical fixation method of AI.

1940- Philips and Lardy developed egg yolk phosphate diluter for preserving fertility and motility of refrigerated bull spermatozoa.

\section{Objective of AI in canines}

AI is generally reserved for valuable purebred dogs that are unable to conceive through natural means due to various problems like

Abnormal vulvar or vaginal conformation Aggression

Male has poor sex drive or weakness or pain when mounting due to arthritis or prostatic disease

Geographic constraints

For some dogs, natural breeding is unsuccessful and artificial methods are required

\section{AI technique}

Begins with collection of semen from male.Semen collection should be performed before the physical exam or any stressful procedures on the stud, or can be booked to another day. ${ }^{4}$ Collection of semen should be prepared in advance, and interval between collections or between the natural mating and collection, should be registered, if the male is regularly used. Ideal intervals between collections are 2 to 5 days, whilst intervals longer than 10 days may result in an increased number of morphological abnormalities and decreased motility. ${ }^{6}$ 
Semen can be used fresh (chilled and used within 24 hours) or frozen.

More processing of semen, less likely it will result in fertilization.

A semen extender fluid can be added to nourish semen (different extenders for fresh or frozen semen are used)

Fresh, chilled, or frozen semen may be used for artificial insemination. Semen is collected from male dogs using an artificial vagina and manual stimulation. Latex products should be avoided because latex has been reported to decrease sperm motility. ${ }^{1}$

Collected sperm should be analyzed for numbers, viability, motility, and morphology. ${ }^{4,5}$ The conception rate is best with fresh semen $(80 \%)$, followed by chilled $(60 \%)$ and frozen $(50 \%$ to $60 \%$ ), but may vary according to the insemination technique used and the skill of the operator. The conception rate also depends on the proper handling of the semen and the fertility of the bitch.

\section{Volume of semen required}

It depends on the size of the female:

Bitches less than $10 \mathrm{lbs}$ need 1.5-3 mls of semen per insemination.

Bitches 10-50 lbs need 3-5 mls of semen

Bitches over $50 \mathrm{lbs}$ need 5-8 mls of semen

\section{Evaluation of semen}

Biological tests:

Normal forward or progressive motility of spermatozoa

Normal morphology of spermatozoa (head, mid-piece and tail)

Normal concentration of live spermatozoa

\section{Physical tests}

Colour: When analysing the colour, one should be aware of the method of collection, as colour varies with the fraction to be analysed and the fact that analysis may been performed on the whole semen or on fractioned semen. The normal colour of whole ejaculate is greyish-white. Pathological colours include: green-greyish typical for the presence of the pus in semen; red or pinkspecific for erythrocytes contamination (haemorrhages from urethra or corpora cavernosa, prostatitis); yellow specific for urine contamination; and brown, if in the presence of blood. $^{8}$

Volume: The volume of the ejaculate may be assessed in the calibrated tubes used for semen collection. It mainly dependents on the size of the dog, the size of the prostate gland, the animal age, the frequency of semen collection, the level of erotisation, and the volume of $3 \mathrm{rd}$ fraction collected.

A decrease of semen volume is observed in cases of benign prostatic hyperplasia, prostatic cysts, inflammatory lesions of prostate and testicles, inflammation of epididymis, vas deferens or urethra and at weak libido. ${ }^{8}$

Biochemical tests: e.g., Fructolysis index: is defined as the amount of fructose utilized by $10^{9}$ spermatozoa in one hour at $37^{\circ} \mathrm{C}$. Greater the metabolic activity of spermatozoa more will be the amount of fructose metabolized in any semen sample.

\section{When to inseminate}

To determine best time based on vaginal cytology or through progesterone levels.

The goal of breeding is to inseminate the bitch four days before ovulation and then 
every two days until the final insemination at two days after ovulation (this results in maximum litter size).

Once prepared, the semen can be deposited either in front of the cervix or just inside the uterus.

Samples deposited just in front of the cervix result in the best success.

Placement of the semen into the proper area of the reproductive tract requires some specialized equipment. (Long pipettes are commercially available)

\section{AI equipments}

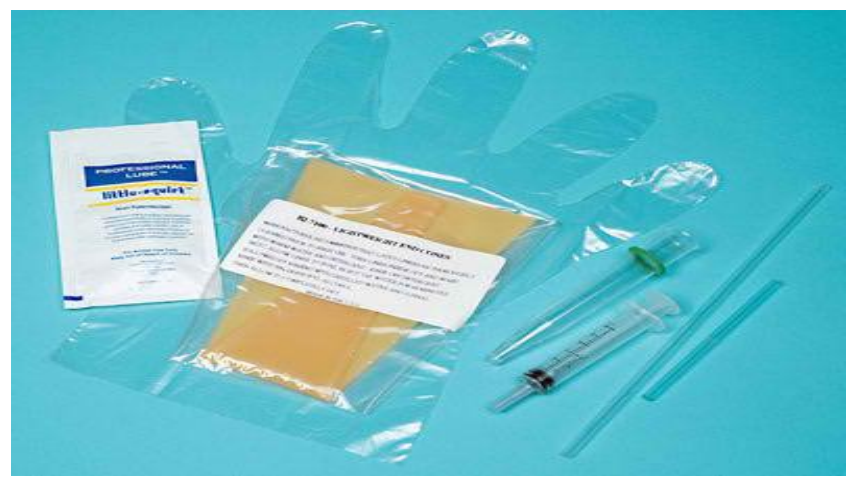

\section{Insemination procedure}

Male should not be around the female.

Hold the female in a standing position.

Draw the appropriate amount of semen into a sterile syringe and attach this to the appropriate pipette.

Pipette is then guided into the upper vagina by a gloved, lubricated finger inserted into the vaginal canal.

Semen is deposited

Syringe is filled with air, and remaining semen is injected after initial push of the syringe.

Pipette is removed

Vaginal wall is stimulated with inserted finger for several minutes

Finger is removed.
Rear of dog is elevated for 5-10 minutes.

Do not push abdomen.

Do not allow dog to squat.

Do not allow to urinate or jump for $30-60$ minutes.

Female can return to normal activity after one hour.

\section{Artificial insemination in the Bitch}

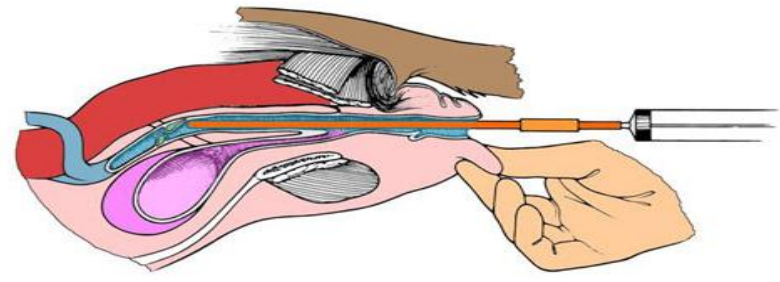

\section{Surgical insemination}

Uterus is exposed, the semen sample is introduced into the uterus by a needle and syringe.

Not the most effective method but may be required.

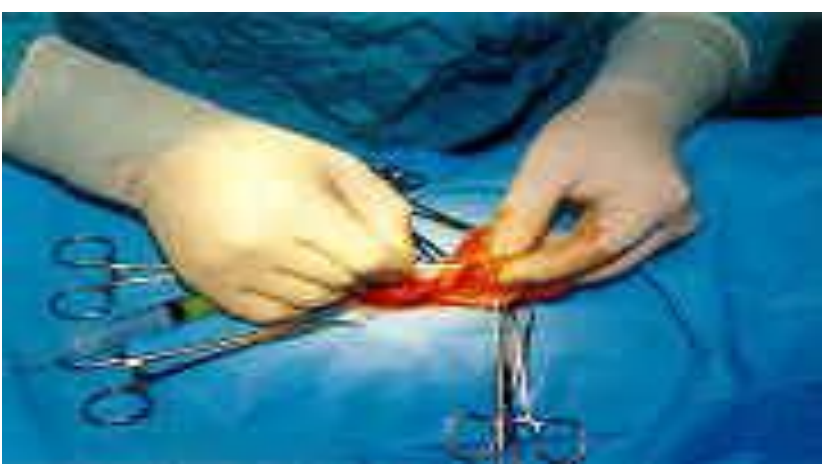

\section{Results of AI in bitches}

Natural breeding there is $80-95 \%$ success rate of pregnancy

With AI using fresh semen, 62-100\% success rate

With AI using chilled semen, 59-80\% success rate

With AI using frozen semen deposited into 
the vagina, $52-60 \%$ success rate

With AI using frozen semen deposited into the uterus, $0-80 \%$ success rate.

\section{Advantages of AI in Dogs}

Artificial insemination allows dogs from all over the world to be able to produce puppies without being shipped anywhere for breeding.

AI allows the semen of the male to be evaluated for fertility and any possible abnormalities

It also prevents any sexually transmitted diseases from being passed from a stud to the bitch or vice versa

Inexperienced dogs can be used to produce offspring without worry about training to mount and breed the bitch

Behavioral objections to natural copulation can also be overcome with artificial insemination

\section{Disadvantages of AI in Dogs}

Frozen semen is relatively less fertile than that of fresh semen hence reduced conception rate

Frozen semen must be stored properly to maintain viability

Labor and training is needed to perform AI than to facilitate natural mating.

\section{References}

1. Athouse GC, Ko JC, Hopkins SM, Evans LE. 1991: Effect of latex and vinyl examination gloves on canine spermatozoal motility. JAVMA 199(2): 227-229.

2. England GC: Artificial insemination, in
Price CJ, Bedford PG, Sutton JB (eds): Fertility and Obstetrics in the Dog. Oxford, UK, Blackwell Science, 1998, pp. 165-172.

3. Farstad W.K. 2010. Artificial insemination in dogs, In BSAVA Manual of Canine and Feline Reproduction and Neonatology, 2nd edition, England G. and von Heimendahl A. (Eds.).British Small Animal Veterinary Association, 978-1-90531.

4. Freshman J L (2002), "Semen collection and evaluation", Clinical Techniques in Small Animal Practice, Vol. 17, pp. 104107.

5. Johnston S.D., Root Kustritz. M.V. Olson P.N.S. 2001Canine and Feline Theriogenology, W.B. Saunders Comp., 978-0-72165-607-6, Philadelphia.

6. Johnston SD, Root Kustritz MV, Olson PN (eds) 2001. Semen collection, evaluation and preservation, in Canine and Feline Theriogenology. Philadelphia, WB Saunders, 2001, pp 287-306.

7. Linde Forsberg. C. 2005. Artificial Insemination. In ESAVS-EVSSAR Course Reproduction in Companion, exotic and laboratory animal, Nantes 1EOF15EOF Reference 5.1.

8. Rita PC, Miranda S and Nizanski W. 2011:Artificial Insemination in Dogs, Artificial Insemination in Farm Animals, Milad Manafi, Intech Open, DOI: 10.5772/20374

9. Sodenberg SF.: 1986: Canine breeding management. Vet Clin North Am 16(3):419-433.

10. Threlfall W: Semen collection and evaluation, in Root Kustritz MV (ed) 2003: Small Animal Theriogenology. St. Louis, Elsevier Science, pp. 97-123.

\section{How to cite this article:}

Jumi Dutta and Joli Dutta. 2020. Artificial Insemination in Canines: A Review. Int.J.Curr.Microbiol.App.Sci. 9(08): 2417-2421. doi: https://doi.org/10.20546/ijcmas.2020.908.276 